\title{
Prevalência de doenças musculoesqueléticas autorreferidas segundo variáveis demográficas e de saúde: estudo transversal de idosos de Goiânia/GO
}

\author{
Prevalence of self-reported musculoskeletal diseases by \\ demographic and health variables: cross-sectional study of \\ elderly of Goiânia/GO
}

\author{
Anna Cássia Fernandes Melo ${ }^{1}$, Adélia Yaeko Kyosen Nakatani², \\ Lilian Varanda Pereira ${ }^{3}$, Ruth Losada de Menezes ${ }^{4}$, Valéria Pagotto ${ }^{5}$
}

\begin{abstract}
Resumo
Objetivo: Estimar a prevalência de doenças musculoesqueléticas autorreferidas por idosos segundo variáveis demográficas, dor, autoavaliação de saúde e quedas. Métodos: Estudo transversal, de base populacional, com amostra probabilística de 934 idosos residentes em Goiânia, em Goiás. Aplicou-se um questionário padronizado e semiestruturado. Considerou-se doença musculoesquelética autorreferida pelo idoso como variável desfecho, enquanto as variáveis de exposição foram demográficas, autoavaliação de saúde, quedas, dor e atividade física. Para análise, foram utilizados os testes qui-quadrado ou Fisher e regressão de Poisson (valor de $p<0,05)$. Resultados: Dos 934 idosos, $62,2 \%$ eram mulheres, com média de 71,4 anos $( \pm 8,3)$. A prevalência de doenças musculoesqueléticas foi de $39,1 \%$ (IC95\% 35,9-42,3), sendo as mais frequentes: osteoporose (24,6\%), artrose (2,9\%), reumatismo $(1,2 \%)$ e artrite $(0,6 \%)$. A prevalência foi superior nas mulheres $(44,7 \% ; p=0,000)$; faixa etária $\geq 80$ anos $(44,7 \%$; $p=0,002)$; autoavaliação de saúde ruim $(55,9 \% ; p=0,000)$; relato de dor $(43,4 \% ; p=0,001)$. Não houve associação entre doenças musculoesqueléticas e quedas $(p=0,671)$ e sedentarismo $(p=0,167)$. Conclusão: Observaram-se elevada prevalência de doenças musculoesqueléticas nos idosos de elevada faixa etária e associação com autoavaliação de saúde ruim e relato de dor, o que sugere intervenções no controle das condições de saúde gerais.
\end{abstract}

Palavras-chave: doenças musculoesqueléticas; idoso; estudos transversais.

\footnotetext{
Abstract

Objective: Estimate the prevalence of self-reported musculoskeletal disorders in elderlies according to demographic variables, pain, self-assessment of health and falls. Methods: Cross-sectional and population-based study with a probabilistic sample of 934 seniors living in Goiania, GO. It was applied a standardized and semi-structured questionnaire. The outcome variable was self-reported musculoskeletal disorders by elderly and exposure were demographic, self-assessment health, falls, pain and physical activity.

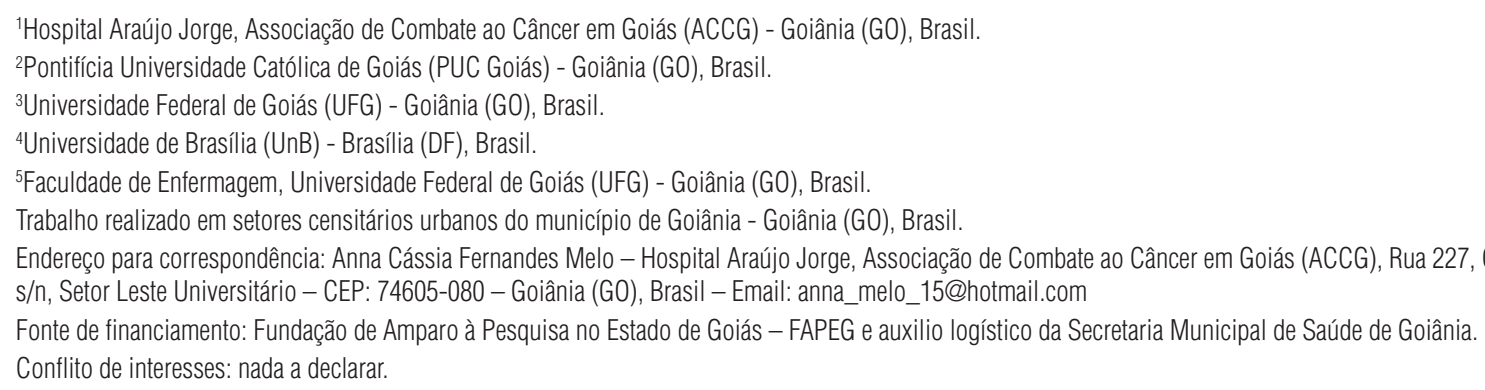


For analysis, we used the Chi-square test or Fisher and Poisson regression (value of $p<0.05$ ). Results: We evaluated 934 elderly, $62.2 \%$ were women, average of 71.4 years $( \pm 8.3)$. The prevalence of musculoskeletal disorders was $39.1 \%(95 \% \mathrm{Cl}-42.335 .9)$, being the most frequent: osteoporosis $(24.6 \%)$, arthritis $(2.9 \%)$, rheumatism $(1.2 \%)$ and arthritis $(0.6 \%)$. The prevalence was higher in women (44.7\%; $p=0.000)$; age $\geq 80$ years $(44.7 \% ; p=0.002)$; self-assessment of bad health $(55.9 \%$; $p=0.000)$; report of pain (43.4\%; $p=0.001)$. There was no association between musculoskeletal disorders and falls $(p=0.671)$ and sedentary $(p=0.167)$. Conclusion: We observed a high prevalence of musculoskeletal disorders in the elderly with high age group which suggests interventions in the control of the general health conditions and pain.

Keywords: musculoskeletal diseases; aged; cross-sectional studies.

\section{INTRODUÇÃO}

O envelhecimento humano contribui para a ocorrência de diminuição de massa óssea, dano às estruturas cartilaginosas, redução da elasticidade dos ligamentos, perda de força muscular e infiltração gordurosa nos tecidos, diminuindo a capacidade destes em manter suas funções normais e podendo levar a doenças como osteoporose, sarcopenia, fratura por trauma leve, osteoartrite e artrite inflamatória ${ }^{1-3}$.

Essas enfermidades, conhecidas como doenças musculoesqueléticas (DME), são a principal causa de incapacidade relacionada à diminuição da mobilidade, principalmente, na faixa etária 65 anos ou mais. Elas podem comprometer a realização de atividades da vida diária (AVD) ${ }^{3}$, bem como, eventualmente, ocasionar dependência e institucionalização, influenciar na autopercepção de saúde e qualidade de vida ${ }^{4-6}$, e implicar em aumento de custos para os serviços ${ }^{7,8}$. Entre as DME, a osteoporose é a mais prevalente mundialmente, apresentando como principais complicações fragilidade óssea, fratura e incapacidade ${ }^{10}$.

Poucos estudos analisaram a prevalência de DME no Brasil. Estudo de revisão sistemática mostrou que as DME mais prevalentes foram artrite ou reumatismo (24,2-37,5\%), osteoartrose (16,6-39,6\%), tendinite $(9,2 \%)$, bursite $(3,8 \%)$, e outras $(10,7 \%)^{11}$. Os fatores que se associam à ocorrência de DME podem ser de caráter intrínseco, como sexo feminino, idade avançada, origem asiática, cor branca, deficiência hormonal, presença de comorbidades, fatores genéticos, fragilidade ou histórico de fratura prévia ${ }^{3}$, fraqueza de membros inferiores, déficit de equilíbrio, diminuição da força de preensão e polifarmácia ${ }^{12}$, e extrínsecos, que são relacionados ao estilo de vida, como baixo peso corpóreo, sedentarismo, tempo de exposição ao tabaco, uso abusivo de álcool e deficiência de vitamina $\mathrm{D}^{3,12}$. A dor também está associada às DME, constituindo-se a principal queixa das pessoas com DME crônicas, promovendo impacto na qualidade de vida, independência e participação social do idoso ${ }^{11}$.

Considerando o aumento da expectativa de vida na atualidade e nos próximos anos no Brasil, as DME têm tendência crescente, pois os idosos constituem o grupo com maior prevalência dessas doenças ${ }^{13,14}$, o que pode levar ao aumento da morbimortalidade, restrição de mobilidade, incapacidade funcional, isolamento social e aumento do risco de quedas ${ }^{15,16}$. Considerando que poucos estudos analisaram essa temática no Brasil, e dadas as consequências das DME para a saúde do idoso, o objetivo deste estudo foi estimar a prevalência de DME autorreferidas por idosos segundo variáveis demográficas, dor, autoavaliação de saúde e quedas.

\section{METODOLOGIA}

Trata-se de um estudo de delineamento transversal, de base populacional, inserido na pesquisa matriz "Situação de saúde da população idosa do município de Goiânia-GO”, vinculado à Rede de Vigilância à Saúde do Idoso no Estado de Goiás (REVISI). Os detalhes metodológicos e de cálculo amostral já foram descritos em publicações prévias ${ }^{17,18}$.

Resumidamente, a amostra foi calculada considerando-se os seguintes parâmetros: população de idosos de Goiânia (7\% do total da população, que era de 1.249 .645 , tendo como ano-base 2007); frequência esperada de $30 \%$ para todos os eventos do inquérito; nível de confiança de 95\%; nível de significância de $5 \%$; frequência esperada de $30 \%$; precisão absoluta de $5 \%$; efeito de delineamento de 1,8 . Com isso, obteve-se um valor amostral de 823 idosos. A esse cálculo foram acrescidos 11\% para possíveis perdas, resultando em 934 idosos, conferindo poder amostral suficiente para as análises do presente estudo.

Para seleção dos idosos, foi realizada amostragem por conglomerados. A área geográfica do estudo foi definida a partir dos Setores Censitários (SC) estabelecidos pelo Instituto Brasileiro de Geografia e Estatística (IBGE). Para a delimitação dos SC, utilizou-se o mapa urbano básico digital de Goiânia, fornecido pela COMDATA (instituição municipal responsável pela construção da malha digital da cidade). Identificaram-se 1.068 SC no município, dos quais 912 eram estritamente urbanos. A média de indivíduos por setor era de 980 pessoas. Considerando 7\% de idosos na população de Goiânia, estimaram-se 16,3 idosos por SC. Dividindo-se o total da amostra $(n=934)$ pelo número de idosos estimados por SC $(17,0)$, calculou-se que seriam necessários $55 \mathrm{SC}$ para a coleta dos dados. Sorteou-se um setor a mais (56) por meio de tabela de números aleatórios criada em sistema eletrônico de randomização. Nesses setores, foram sorteados o quarteirão e a esquina para o início da coleta. A partir da esquina sorteada, a primeira residência foi visitada. Caso não houvesse idoso, a entrevistadora deslocava-se para o próximo domicílio até identificá-lo. Na ocorrência de mais de um idoso residindo no domicílio, todos foram entrevistados. 
Quando o número de domicílios residenciais do setor não era suficiente para completar a amostra, outro SC já estava sorteado; então o entrevistador prosseguia até aproximar ou completar o total estimado de idosos. Foram incluídos: indivíduos que possuíam idade igual ou superior a 60 anos, que residiam na área urbana de Goiânia e que eram moradores da residência da entrevista. Foram excluídos os idosos que não responderam à pergunta sobre DME.

Os dados foram coletados entre os meses de dezembro de 2009 e abril de 2010 por entrevistadores previamente treinados para a aplicação do questionário, após a aplicação do teste-piloto que foi realizado nos meses de outubro e novembro de 2009 com população de 50 idosos de um SC não sorteado para a coleta dos dados.

Os instrumentos foram preenchidos e entregues em uma secretaria central para conferência e identificação de possível inconsistência dos dados. Quando alguma incongruência de dados era identificada, o entrevistador retornava ao domicílio e corrigia os registros antes de seu envio ao banco de dados eletrônico. O controle de qualidade dos dados foi realizado por pesquisadores e técnicos treinados que supervisionaram todos os questionários aplicados logo após a entrevista e antes de sua inserção no banco de dados. Já o fomento de dados no sistema foi feito por pares. Após o término do processo de digitação dos dados, o banco foi integralmente conferido para reavaliação de possíveis incongruências que poderiam ter persistido.

A variável desfecho deste estudo foi DME autorreferida, avaliada por meio da seguinte pergunta: "Quais doenças o médico já disse que o(a) Sr. (a) têm?”. Todas as respostas referentes às DME foram agrupadas de acordo com a Classificação Internacional de Doenças (CID) em uma única variável dependente: DME.

As variáveis de exposição foram demográficas: (sexo e faixa etária), quedas, dor autorreferida e autoavaliação do estado de saúde. A ocorrência de quedas foi avaliada por meio da pergunta: “ $O(a)$ Sr. (a) caiu no último ano?”. Para autoavaliar sua saúde, os idosos responderam à seguinte questão: "O que o(a) Sr. (a) acha do seu estado de saúde no último mês?”. As opções de resposta foram categorizadas em: bom/muito bom; regular; ruim/muito ruim. A dor existente há seis meses ou mais foi avaliada pelo autorrelato.

Os dados foram digitados no programa Excel ${ }^{\circledR}$ for Windows 2003-2007 e analisados no software Stata ${ }^{\circledR}$, versão 11.0. As variáveis foram primeiramente analisadas de forma descritiva por meio de frequência absoluta, relativa, média e desvio-padrão. Para a análise de associação entre as variáveis independentes e o desfecho, foi utilizado o teste qui quadrado ou Fisher, adotando-se nível de significância de 5\%. A magnitude da associação foi estimada pela razão de prevalência (RP), considerando-se nível de significância de $5 \%(\mathrm{p}<0,05)$.

A pesquisa foi aprovada pelo Comitê de Ética em Pesquisa da Universidade Federal de Goiás sob o protocolo n 050/2009, conforme as normas nacionais em ética em pesquisa vigente sobre pesquisas envolvendo seres humanos. Os participantes consentiram em participar mediante assinatura do Termo de Consentimento Livre e Esclarecido (TCLE).

\section{RESULTADOS}

Foram entrevistados 934 idosos, sendo $62,2 \%(n=581)$ do sexo feminino, $48,1 \%$ na faixa etária de 60-69 anos, com idade média de 71,4 anos $( \pm 8,3), 57,9 \%$ com renda inferior a um salário mínimo, $48,2 \%$ com nível de escolaridade na fase do ensino primário, e $49,1 \%$ casados.

A prevalência de DME foi de 39,1\% (IC95\% 35,9-42,3) (Figura 1). As principais doenças autorreferidas foram: osteoporose $(24,6 \%)$, artrose $(2,9 \%)$, reumatismo $(1,2 \%)$ e artrite $(0,6 \%)$. Do total, $9,8 \%$ das pessoas não souberam relatar o tipo de DME que referiram ter (Tabela 1 ).

Observou-se maior prevalência de DME entre as mulheres (49,6\%). A probabilidade de apresentar DME foi 2,3 vezes maior entre as mulheres do que nos homens ( $\mathrm{RP}=2,3$; IC95\% 1,8-2,8). Em relação à faixa etária, a prevalência foi superior nos idosos acima de 70 anos (superior a 40\%), e as medidas de efeito demonstraram que a prevalência de DME nas faixas etárias mais altas pode ser $13 \%$ maior em relação à faixa etária mais jovem (RP=1,3; IC95\% 1,1-1,7) (Tabela 2).

Analisando a prevalência conforme as variáveis de exposição (Tabela 3), observa-se que a prevalência de DME foi superior em idosos que autoavaliaram sua saúde como ruim (55,9\%),

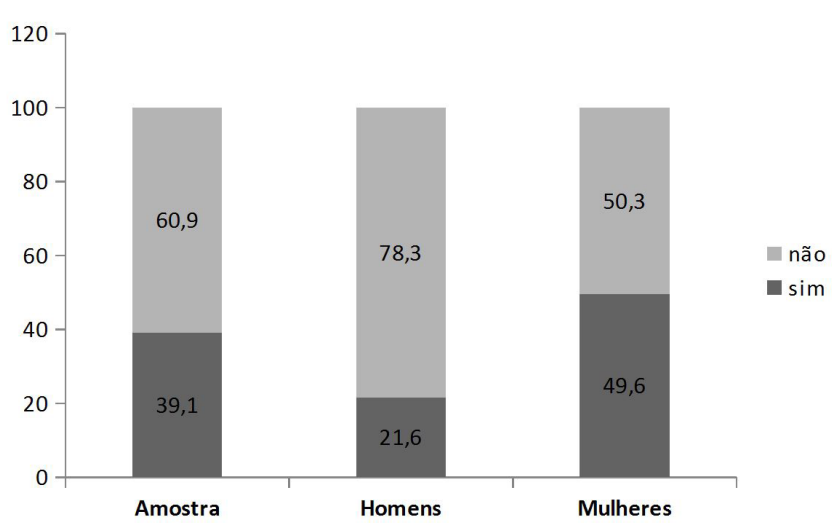

Figura 1. Prevalência de doenças musculoesqueléticas conforme sexo em idosos do município de Goiânia, Goiás, em 2010 (n=934)

Tabela 1. Prevalência de doenças musculoesqueléticas (DME) autorreferidas por idosos do município de Goiânia, Goiás, em 2010 $(n=934)$

\begin{tabular}{lcc}
\multicolumn{1}{c}{ DME } & n (\%) & IC95\% \\
Osteoporose & $229(24,6)$ & $21,8-27,5$ \\
Artrose & $25(2,7)$ & $1,7-3,9$ \\
Reumatismo & $11(1,2)$ & $0,5-2,1$ \\
Artrite & $6(0,6)$ & $0,2-1,4$ \\
Outros & $94(9,8)$ & $8,2-12,2$ \\
\hline
\end{tabular}


Tabela 2. Prevalência de doenças musculoesqueléticas (DME) e razão de prevalência (RP) conforme sexo e faixa etária em idosos no município de Goiânia, Goiás, em 2010 (n=934)

\begin{tabular}{lcccc}
\multicolumn{1}{c}{ Variáveis } & N (\%) & Prevalência de DME (\%) & RP (IC\%) & 0,000 \\
Sexo & & & & $\boldsymbol{p}^{*}$ \\
Feminino & $581(62,2)$ & 49,7 & $2,3(1,8-2,8)$ & 0,002 \\
Masculino & $353(37,8)$ & 21,6 & 1,0 \\
Faixa etária (1) & & & 1,0 \\
60-69 anos & $451(48,3)$ & 33,2 & $1,3(1,1-1,6)$ \\
$70-79$ anos & $310(33,2)$ & 44,5 & $1,3(1,1-1,7)$ \\
$\geq 80$ anos & $173(18,5)$ & 44,8 & \\
\hline
\end{tabular}

${ }^{\star}$ Qui-quadrado

Tabela 3. Prevalência de doenças musculoesqueléticas (DME) e razão de prevalência (RP) conforme condições de saúde em idosos no município de Goiânia, Goiás, em 2010 (n=934)

\begin{tabular}{|c|c|c|c|c|}
\hline Variáveis & $\mathrm{N}(\%)$ & Prevalência de DME (\%) & RP (IC\%) & $p^{*}$ \\
\hline Autoavaliação de saúde & & & & 0,000 \\
\hline Muito bom/bom & $396(44,5)$ & 30,8 & 1,00 & \\
\hline Regular & $391(44)$ & 44,0 & $1,4(1,2-1,7)$ & \\
\hline Ruim/muito ruim & $102(11,5)$ & 55,9 & $1,8(1,4-2,3)$ & \\
\hline Quedas no último ano & & & & 0,671 \\
\hline Sim & $318(34,8)$ & 39,3 & $1,0(0,9-1,2)$ & \\
\hline Não & $595(65,2)$ & 37,9 & 1,0 & \\
\hline Dor & & & & 0,001 \\
\hline Sim & $543(59,4)$ & 43,4 & $1,3(1,1-1,6)$ & \\
\hline Não & $371(40,6)$ & 32,6 & 1,0 & \\
\hline Atividade física & & & & 0,167 \\
\hline Sim & $287(31,2)$ & 35,5 & 1,0 & \\
\hline Não & $632(68,8)$ & 40,4 & $1,1(0,9-1,4)$ & \\
\hline
\end{tabular}

${ }^{*}$ Qui-quadrado

que referiram dor (43,4\%), quedas (39,3\%) e naqueles que não praticavam atividade física $(40,4 \%)$. Foram associadas às DME: autoavaliação do estado de saúde regular ( $\mathrm{RP}=1,4$; IC95\% $1,2-1,7)$ e ruim/muito ruim ( $\mathrm{RP}=1,8$; IC95\% 1,4-2,3), além de relato de dor $(\mathrm{RP}=1,3$; IC95\% 1,1-1,6).

\section{DISCUSSÃO}

A prevalência total de DME nos idosos deste estudo foi elevada $(39,1 \%)$, com destaque para osteoporose (24,6\%). Prevalência inferior foi observada no Canadá 4 , com 18,5\% de idosos com artrite, $20,5 \%$ com problemas na coluna e 30\% referindo alguma DME em geral. Quanto à prevalência de osteoporose, resultados inferiores foram encontrados na China ${ }^{7}(15,7 \%)$ e na Noruega ${ }^{19}$ $(19,6 \%)$, mas semelhantes em Portugal ${ }^{8}(25,3 \%)$. Já no Brasil ${ }^{20}$, a Pesquisa Nacional por amostra de Domicílio (PNAD) identificou prevalência decrescente de artrite, com $37,5,27,3$ e $24,2 \%$, respectivamente, nos anos de 1998, 2003 e 2008.

Outro estudo nacional ${ }^{11}$ apontou estimativas de prevalência de DME de 9 a $40 \%$ na população idosa. Já em uma pesquisa de base populacional realizada no Rio Grande do $\mathrm{Sul}^{21}$, a artrite foi relatada por $43,1 \%$ dos idosos. Observa-se elevada prevalência de DME em estudos brasileiros quando comparados aos internacionais.
Um estudo feito em São Paulo ${ }^{22}$ evidenciou prevalência de $21,3 \%$ de osteoporose em mulheres menopausadas com idade superior a 50 anos. Recentemente, uma pesquisa ${ }^{23} \mathrm{em}$ São Paulo com idosos com idade de 80 anos ou mais mostrou prevalência de artrite/artrose de 26 e $33 \%$, alguma doença na coluna com 12 e 19\%, e osteopenia/osteoporose de 71 e $49 \%$ em homens e mulheres, respectivamente.

A maior predominância de DME em mulheres neste estudo se justifica, primeiramente, ao fato da maior expectativa de vida para o sexo feminino, o que faz com que apresentem maior prevalência de doenças em relação aos homens ${ }^{7}$. Estudos prévios realizados no Brasil ${ }^{22,24,25}$, no Paquistão ${ }^{26}$ e no Irã ${ }^{27}$ mostraram que a prevalência de osteoporose nas mulheres é quase o dobro em relação aos homens. Outra justificativa é porque as mulheres apresentam maior velocidade de perda óssea após a menopausa devido à diminuição hormonal significativa nos níveis de estrogênio?.

Houve uma maior prevalência de DME na faixa etária mais idosa. Esse achado era esperado porque os tecidos musculoesqueléticos apresentam, com o envelhecimento, crescente fragilidade óssea, dano às estruturas cartilaginosas, redução da elasticidade dos ligamentos, perda de força muscular e infiltração gordurosa nos tecidos, condições que em faixas etárias avançadas estão 
intensificadas e podem levar à $\mathrm{DME}^{1-3,12}$. Entretanto, um estudo de base populacional realizado em Portugal $^{8}$ evidenciou uma associação entre reumatismo e DME maior em idosos jovens, entre 60 e 70 anos, sugerindo que há uma tendência de aumento de DME já nessa faixa etária.

A presença de DME foi associada a uma autoavaliação de saúde ruim/muito ruim. A autoavaliação do estado de saúde é um indicador subjetivo da percepção do indivíduo sobre a própria saúde, que expressa aspectos físicos, emocionais, de bem-estar e de satisfação com a própria vida ${ }^{28}$. Essa associação foi também encontrada em outro estudo desenvolvido em Goiânia com idosos usuários do SUS ${ }^{29}$. Tanto a presença de uma DME, como os seus sintomas podem trazer limitações e falta de autonomia para o idoso executar suas tarefas, o que, por consequência, pode gerar uma percepção negativa de sua saúde geral ${ }^{29,30}$.

A associação entre dor e DME é um evento esperado, uma vez que a dor é uma das principais queixas das pessoas com essas doenças, o que é coerente em estudos prévios ${ }^{11,12}$. Um trabalho prospectivo observacional ${ }^{31}$ identificou que a dor foi proporcionalmente aumentada conforme a idade e a perda da mobilidade por DME autorrelatadas. Neste estudo, não foi abordado o controle da dor nem a adesão a medicamentos, porém, outra pesquisa com idosos em um centro de tratamento medicamentoso para dor crônica ${ }^{32}$ identificou que a maioria dos agentes causadores da dor crônica era as DME. Além disso, outro estudo ${ }^{33}$ revelou ser frequente na população idosa com alguma DME a automedicação para o controle dos sintomas.

As DME associadas à dor também podem interferir na locomoção desses idosos, propiciando desequilíbrio, restrição de mobilidade, e, consequentemente, aumentando o risco de queda ${ }^{12}$. Esse processo é atribuído às perdas progressivas de equilíbrio e de alterações na massa muscular e óssea, que ocorrem com o processo de envelhecimento. Um estudo de base populacional ${ }^{2}$ avaliou a ocorrência de quedas em 1.520 idosos por 12 meses. Embora a prevalência geral tenha sido menor do que neste estudo (6,5\%), evidenciou-se que, entre outros fatores, sexo feminino ( $\mathrm{RP}=2,4 ; 1,5-3,9)$, idade maior ou igual a 80 anos $(\mathrm{RP}=2,5 ; 1,6-3,9)$, reumatismo/artrite/artrose $(\mathrm{RP}=1,6$; $1-2,5)$ e osteoporose ( $\mathrm{RP}=1,7 ; 1,2-2,5)$ são preditores de queda.

Embora neste estudo não tenha sido significativa a associação com presença de DME, algumas pesquisas ${ }^{6,34}$ apontam a atividade física como fator protetor tanto na prevenção quanto na morbidade provocada por DME, principalmente incapacidade e dor. Em um estudo prospectivo envolvendo idosos com alguma $\mathrm{DME}^{6}$, exercícios de fisioterapia implicaram na redução de dor autorrelatada e melhora gradual do desempenho.

Pode-se destacar como limitações deste estudo o tipo de delineamento transversal, que impossibilita inferir causalidade, não sendo possível afirmar em qual momento do tempo ocorreram a exposição e o desfecho. Outra limitação refere-se à natureza autorreferida da presença de DME. Entretanto, os entrevistadores foram treinados para garantir a qualidade e a fidedignidade das informações aqui apresentadas.

Os resultados desta pesquisa demonstraram elevada prevalência de idosas com DME, sendo a osteoporose a mais prevalente. O autorrelato de dor e a autoavaliação negativa da saúde foram associadas à ocorrência de DME e sinalizam a necessidade de monitoramento dessas condições, uma vez que podem interferir na limitação funcional do idoso devido à diminuição da mobilidade. Considerando o aumento da expectativa de vida na atualidade e que as DME são prevalentes, recomenda-se que ações de prevenção sejam reforçadas na atenção primária à saúde, nível de atenção em que os idosos têm maior acesso aos profissionais de saúde. Espera-se também que estes dados contribuam para subsidiar políticas e ações de saúde locais e possam ser utilizados na implantação de projetos e no aumento de informação para adoção de atitudes preventivas.

\section{AGRADECIMENTOS}

Os autores gostariam de agradecer o apoio financeiro da Fundação de Amparo à Pesquisa no Estado de Goiás (FAPEG) e o auxílio logístico da Secretaria Municipal de Saúde (SMS), sem os quais não seria possível dar prosseguimento a este estudo.

\section{REFERÊNCIAS}

1. Gheno R, Cepparo JM, Rosca CE, Cotten A. Musculoskeletal disorders in the elderly. J Clin Imaging Sci. 2012;2(3):39. PMid:22919553.

2. Rodrigues IG, Fraga GP, Barros MBA. Quedas em idosos: fatores associados em estudo de base populacional. Rev Bras Epidemiol. 2014;17(3):705-18. PMid:25272263. http://dx.doi.org/10.1590/1809-4503201400030011.

3. Curtis E, Litwic A, Cooper C, Dennison E. Determinants of muscle and bone aging. J Cell Physiol. 2015;230(11):2618-25. PMid:25820482. http:// dx.doi.org/10.1002/jcp. 25001 .

4. Jover JA, Lajas C, Leon L, Carmona L, Serra JA, Reoyo A, et al. Incidence of physical disability related to musculoskeletal disorders in the elderly: results from a primary care-based registry. Arthritis Care Res. 2015;67(1):89-93. PMid:25074816. http://dx.doi.org/10.1002/acr.22420.

5. Slater M, Perruccio AV, Badley EM. Musculoskeletal comorbidities in cardiovascular disease, diabetes and respiratory disease: the impact on activity limitations; a representative population-based study. BMC Public Health. 2011;11(77):1-7. PMid:21291555.

6. Fritz JM, Hunter SJ, Tracy DM, Brennan GP. Utilization and clinical outcomes of outpatient physical therapy for medicare beneficiaries with musculoskeletal conditions. Phys Ther. 2011;91(3):330-45. PMid:21233306. http://dx.doi.org/10.2522/ptj.20090290.

7. Lin X, Xiong D, Peng Y, Sheng Z, Wu X-Y, Wu X-P, et al. Epidemiology and management of osteoporosis in the People's Republic of China: current perspectives. Clin Interv Aging. 2015;10:1017-33. PMid:26150706.

8. Rodrigues AM, Gouveia N, Costa LP, Eusébio M, Ramiro S, Machado $\mathrm{P}$, et al. EpiReumaPt: the study of rheumatic and musculoskeletal diseases 
in Portugal: a detailed view of the methodology. Acta Reumatol Port. 2015;40(2):110-24. PMid:26219965.

9. Lupsa BC, Insogna K. Bone health and osteoporosis. Endocrinol Metab Clin North Am. 2015;44(3):517-30. PMid:26316240. http://dx.doi.org/10.1016/j. ecl.2015.05.002.

10. Baccaro LF, Conde D, Costa-Paiva L, Pinto-Neto AM. The epidemiology and management of postmenopausal osteoporosis: a viewpoint from Brazil. Clin Interv Aging. 2015;10:583-91. PMid:25848234. http://dx.doi. org/10.2147/CIA.S54614.

11. Miranda VS, Carvalho VBF, Machado LAC, Dias JMD. Prevalence of chronic musculoskeletal disorders in elderly Brazilians: a systematic review of the literature. BMC Musculoskelet Disord. 2012;82(13):1-10. PMid:22642899.

12. Bello AI, Ababio E, Antwi-Baffoe S, Seidu MA, Adjei DN. Pain, range of motion and activity level as correlates of dynamic balance among elderly people with musculoskeletal disorder. Ghana Med J. 2014;48(4):214-8. PMid:25709137. http://dx.doi.org/10.4314/gmj.v48i4.8.

13. Mata MS, Costa FA, Souza TO, Mata NAS, Pontes JF. Dor e funcionalidade na atenção básica à saúde. Cien Saude Colet. 2011;16(1):221-30. PMid:21180830. http://dx.doi.org/10.1590/S1413-81232011000100025.

14. Carvalho T, Mozerle A, Ulbrich AZ, Andrade AL, Moraes EEC, Mara LS. Avaliação do sistema locomotor de pacientes de programas de reabilitação cardiopulmonar e metabólica. Arq Bras Cardiol. 2010;95(2):258-63. PMid:20585734. http://dx.doi.org/10.1590/S0066-782X2010005000083.

15. Viana JU, de Oliveira MC, Magalhães TV. Quedas intra-hospitalares na Santa Casa de Belo Horizonte MG são adequadamente relatadas? Fisioterapia e Pesquisa. 2011;18(1):72-8. http://dx.doi.org/10.1590/S180929502011000100013

16. Tomita Y, Arima K, Kanagae M, Okabe T, Mizukami S, Nishimura $\mathrm{T}$, et al. Association of physical performance and pain with fear of falling among community-dwelling japanese women aged 65 years and older. Medicine. 2015;94(35):1-2. PMid:26334906. http://dx.doi.org/10.1097/ MD.0000000000001449.

17. Vera I, Lucchese R, Nakatani AY, Pagotto V, Montefusco SR, Sadoyama. Funcionalidade familiar em longevos residentes em domicílio. Rev Bras Enferm. 2015;68(1):68-75. PMid:25946497. http://dx.doi.org/10.1590/00347167.2015680110p.

18. Pimentel WRT, Pagotto V, Nakatani AYK, Pereira LV, Menezes RL. Quedas e qualidade de vida: associação com aspectos emocionais em idosos comunitários. Geriatr Gerontol Aging. 2015;9(2):42-8. http://dx.doi. org/10.5327/Z2447-2115201500020002.

19. Leiknes KA, Finset A, Moum T. Commonalities and differences between the diagnostic groups: Current somatoform disorders, anxiety and/or depression, and musculoskeletal disorders. J Psychosom Res. 2010;68(5):43946. PMid:20403502. http://dx.doi.org/10.1016/j.jpsychores.2010.02.003.

20. Lima-Costa MF, Matos DL, Camargos VP, Macinko J. Tendências em dez anos das condições de saúde de idosos brasileiros: evidências da Pesquisa Nacional por Amostra de Domicílios (1998, 2003, 2008). Cien Saude Colet. 2011;16(9):3689-96. PMid:21987313. http://dx.doi.org/10.1590/ S1413-81232011001000006.

21. Blay SL, Fillenbaum GG, Andreoli SB, Gastal FL. Prevalence and concomitants of arthritis in the elderly in Rio Grande do Sul, Brazil. PLoS One. 2012;7(9):17. PMid:23028995. http://dx.doi.org/10.1371/journal.pone.0045418.
22. Baccaro LF, Machado VSS, Costa-Paiva L, Sousa MH, Osis MJ, PintoNeto AM. Factors associated with osteoporosis in Brazilian women: a population-based household survey. Arch Osteoporos. 2013;8(1-2):138. PMid:23575503. http://dx.doi.org/10.1007/s11657-013-0138-z.

23. Santos VR, Gobbo LA, Christofaro DGD, Gomes IC, Mota J, Gobbi S, et al. Osteoarticular diseases and physical performance of Brazilians over 80 years old. Cien Saude Colet. 2016;21(2):423-30. PMid:26910150. http:// dx.doi.org/10.1590/1413-81232015212.16032015.

24. Pinheiro MM, Reis No ET, Machado FS, Omura F, Yang JHK, Szejnfeld J, et al. Risk factors for osteoporotic fractures and low bone density in pre and postmenopausal women. Rev Saude Publica. 2010;44(3):479-85. PMid:20549019. http://dx.doi.org/10.1590/S0034-89102010000300011.

25. Buttros DAB, Nahas-Neto J, Nahas EAP, Cangussu LM, Barral BCR, Kawakami MS. Fatores de risco para osteoporose em mulheres na pósmenopausa do sudeste brasileiro. Rev Bras Ginecol Obstet. 2011;33(6):295302. PMid:21877019. http://dx.doi.org/10.1590/S0100-72032011000600006.

26. Habib S, Iqbal R, Shahid M, Habib A. Growing prevalence of osteoporosis in Pakistan: call for action. J Pak Med Assoc. 2015;65(2):230-1. PMid:25842568.

27. Mohammadi Z, Keshtkar A, Fayyazbakhsh F, Ebrahimi M, Amoli MM, Ghorbani M, et al. Prevalence of osteoporosis and vitamin D receptor gene polymorphisms (FokI) in an Iranian general population based study (Kurdistan) (IMOS). Med J Islam Repub Iran. 2015;29:238. PMid:26793629.

28. Pavão ALB, Werneck GL, Campos MR. Autoavaliação do estado de saúde e a associação com fatores sociodemográficos, hábitos de vida e morbidade na população: um inquérito nacional. Cad Saude Publica. 2013;29(4):723-34. PMid:23568302. http://dx.doi.org/10.1590/S0102-311X2013000800010.

29. Pagotto V, Nakatani AYK, Silveira EA. Fatores associados à autoavaliação de saúde ruim em idosos usuários do Sistema Único de Saúde. Cad Saude Publica. 2011;27(8):1593-602. PMid:21877007. http://dx.doi.org/10.1590/ S0102-311X2011000800014.

30. Borim FSA, Barros MBA, Neri AL. Autoavaliação da saúde em idosos: pesquisa de base populacional no Município de Campinas, São Paulo, Brasil. Cad Saude Publica. 2012;28(4):769-80. PMid:22488322. http:// dx.doi.org/10.1590/S0102-311X2012000400016.

31. Shah RC, Buchman AS, Boyle PA, Leurgans SE, Wilson RS, Andersson GB, et al. Musculoskeletal pain is associated with incident mobility disability in community-dwelling elders. J Gerontol A Biol Sci Med Sci. 2011;66(1):82-8. PMid:20966101. http://dx.doi.org/10.1093/gerona/glq187.

32. Markotic F, Cerni Obrdalj E, Zalihic A, Pehar R, Hadziosmanovic Z, Pivic $\mathrm{G}$, et al. Adherence to pharmacological treatment of chronic nonmalignant pain in individuals aged 65 and older. Pain Med. 2013;14(2):247-56. PMid:23368967. http://dx.doi.org/10.1111/pme.12035.

33. Driban JB, Boehret SA, Balasubramanian E, Cattano NM, Glutting J, Sitler MR. Medication and supplement use for managing joint symptoms among patients with knee and hip osteoarthritis: a cross-sectional study. BMC Musculoskelet Disord. 2012;13(1):47. PMid:22458305. http://dx.doi. org/10.1186/1471-2474-13-47.

34. Cawthon PM, Blackwell TL, Cauley JA, Ensrud KE, Dam T, Harrison SL, et al. Objective assessment of activity, energy expenditure, and functional limitations in older men: the osteoporotic fractures in men study. J Gerontol A Biol Sci Med Sci. 2013;68(12):1518-24. PMid:23682162. http://dx.doi. org/10.1093/gerona/glt054.

Recebido em: Nov. 01, 2016 Aprovado em: Mar. 30, 2017 